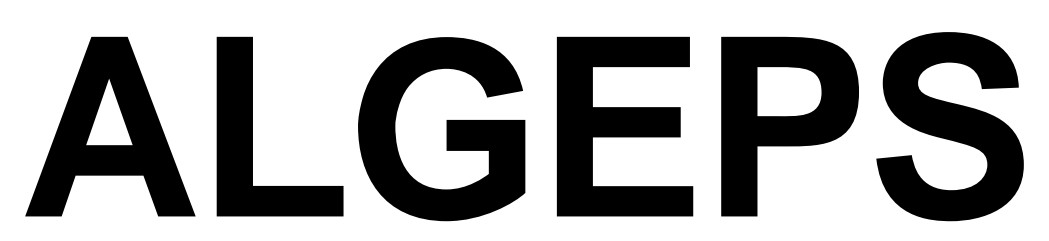

REVISTA DE GEOLOGIA, SÈRIE B no 578 - Gener del 2012

ISSN $1132-7014$

D.L.B. 28.178 - 92

10 pàgines

\begin{abstract}
RECORRIDO GEOLÓGICO Y MINERO DESDE LECERA A MONEVA, MOYUELA, PLENAS y AZUARA, A TRAVÉS DEL PATRIMONIO GEOLÓGICO Y MINERO DE LAS COMARCA DEL CAMPO DE BELCHITE
\end{abstract}

Josep M. Mata-Perelló

Aquest recorregut va ésser experimentat amb docents el dia 1 DE MAIG DEL 2010 


\section{RECORRIDO GEOLÓGICO Y MINERO DESDE LECERA A MONEVA, MOYUELA, PLENAS y AZUARA, A TRAVÉS DEL PATRIMONIO GEOLÓGICO Y MINERO DE LAS COMARCA DEL CAMPO DE BELCHITE}

Josep M. Mata- Perelló

\section{ADVERTENCIAS PREVIAS}

Como en otros recorridos de RECONOCIMIENTO GEOLÓGICO (o de RECONOCIMIENTO GEOLÓGICO Y MINERO), el recorrido se compondrá de diversas PARADAS. En este caso serán seis.

Por otra parte, habrá que tener en cuenta, en todo momento, especialmente antes de empezar los recorridos de los diferentes tramos, el estado de los caminos y carreteras, por donde transitará el recorrido. Al respecto, cabe decir que prácticamente todos estos tramos se halla en buenas condiciones.

Finalmente, como ya hacemos en otros recorridos similares, queremos decir que hace falta tener un cuidado muy especial en el respeto a la naturaleza, a lo largo de todo el recorrido del itinerario, y también fuera de él.

\section{BREVE INTRODUCCIÓN GEOLÓGICA}

El recorrido de este itinerario, se desarrollará exclusivamente por una de las tres unidades geológicas que constituyen el suelo y el subsuelo de Aragón. Concretamente por la Depresión Geológica del Ebro (en donde se iniciarla y finalizara el recorrido del itinerario). Así, a lo largo de todo de todo el recorrido del itinerario, se irán encontrando afloramientos cenozoicos, en su mayoría de carácter arcilloso y calcolutítico, pertenecientes al Mioceno. De entre estos materiales, cabe destacar los afloramientos carbonatados y los yesosos, explotados en diversos lugares del recorrido. Asimismo, a menudo, encontraremos afloramientos de los materiales detríticos cuaternarios, por encima de los anteriores

\section{BREVE INTRODUCCIÓN GEOGRÁFICA}

El recorrido del presente itinerario se efectuará casi exclusivamente por una comarca aragonesa, la del Campo de Belchite. Así, se iniciará en la población de Lecera, para terminar en las cercanías de Azuara. Sin embargo, efectuaremos una 
pequeña entrada en el municipio de Muniesa, perteneciente a la comarca de las Cuencas Mineras.

En este recorrido, se circulará por los términos municipales de: Lecera, Moneva, Moyuela, Plenas y Azuara, todos ellos de la comarca del Campo de Belchite. También pasaremos por el municipio de Muniesa (de la comarca turolense de Cuencas Mineras).

\section{OBJETIVOS GENERALES DE ESTE ITINERARIO}

En este itinerario, los objetivos generales que se han de conseguir, se pueden concretar en los siguientes aspectos:

1.- Estudio y reconocimiento de los materiales cenozoicos (en buena parte del Mioceno) situados en la Depresión Geológica del Ebro, que iremos encontrando a lo largo del recorrido del itinerario, entre las inmediaciones de Lecera y las de la población de Azuara.

2.- Visión de algunas de las antiguas explotaciones encontradas a lo largo del recorrido del itinerario. En concreto, nos centraremos en varias explotaciones de gravas, situadas en Lécera, Plenas y en Azuara.

3.- Observación del impacto producido por las actividades mineras. I si se da el caso, de las restauraciones realizadas para paliar este impacto.

4.- Visión de los diferentes lugares directamente relacionados con el Patrimonio Geológico y Minero que iremos encontrando a lo largo del recorrido del presente itinerario. Por lo que concierne al Patrimonio Geológico veremos el de los Cantiles del río Cámaras, en Azuara. En relación con el Patrimonio Minero, no destacaremos ninguno.

\section{ANTECEDENTES BIBLIOGRÁFICOS}

En relación con este itinerario, no conocemos ningún antecedente, relativo a otro itinerario que discurra por este lugar. En este sentido, este itinerario constituye un antecedente, si no estamos equivocados.

Por otra parte, haremos mención de algunos trabajos, de carácter geológico generalista, que corresponden a los trabajos del IGME (1972, 1974 y 1975), relativos al Mapa Geológico de España (a Escala 1.200.000), al Mapa Metalogenético de España y al Mapa de Rocas Industriales de España

Con respecto a las mineralizaciones que iremos encontrando, mencionaremos los trabajos de: CALVO (2001), CALVO et altri (1988); MAESTRE (1845); así como nuestros trabajos: MATA-PERELLÓ (1987 y 1998). 
También mencionaremos el trabajo de PRAMES (2005) dedicado a la comarca del Campo de Belchite. Así como el del GOBIERNO DE ARAGÓN (2001), dedicado a los Puntos de Interés Geológico de Aragón.

Finalmente, diremos que todos estos trabajos (así como otros que ahora no hemos aludido), figurarán mencionados, por orden alfabético, en el apartado dedicado a las REFERENCIAS BIBLIOGRÁFICAS.

\section{RECORRIDO DEL ITINERARIO}

Este recorrido se iniciará en las inmediaciones del pueblo de Lecera. En torno a ese pueblo se efectuará la primera parada.

Luego, el recorrido se dirigirá hacia el Sur por la carretera de Muniesa, hasta encontrar el desvío que conduce a la población de Moneva, seguidamente se continuará hacia Moyuela, para ir luego hacía Plenas, en donde se realizará una nueva parada. Antes se habran realizados otras dos paradas: una en Ventas de Muniesa (del municipio turolense de Muniesa) y otra en Moneva.

Posteriormente, el recorrido se desplazará hacia la población de Azuara. En este último tramo se realizaran diversas paradas, finalizando el recorrido en la población acabada de mencionar.

\section{DESCRIPCIÓN DEL ITINERARIO}

Como de costumbre, haremos una serio de PARADAS (o ESTACIONES), en donde se realizaran diversas explicaciones en torno a las características del lugar en donde se halla la PARADA.

Por otra parte, en ellas haremos mención del término municipal dónde se encuentran, así como del número del "Mapa Topográfico Nacional (a escala 1:50.000, que indicaremos entre paréntesis. Así, ahora (en este recorrido) utilizaremos solamente las hojas: concretamente las 439 (o de Azuara) y 440 (o de Belchite), 466 (o de Moyuela) y 467 (o de Muniesa).

Así, la relación ordenada de las paradas que constituyen el recorrido de este itinerario, es la siguiente:

PARADA 1. GRAVERA DE LECERA, (término municipal del Lecera, comarca del Campo de Belchite). (Hoja 440).

El recorrido del presente itinerario lo iniciaremos en las inmediaciones de la localidad de Lécera. Concretamente en el desvío al pueblo, desde la carretera que procede de Albalate del Arzobispo, a menos de 0’5 al SE del pueblo. 
Este lugar, como el resto de todo el recorrido del itinerario, se encuentra situado en la Depresión Geológica del Ebro. Así, por doquier veremos afloramientos de los materiales miocénicos que la rellenas. Y también de los materiales detríticos cuaternarios que se superponen a los anteriores.

Precisamente, en este lugar hay un afloramiento de los materiales cuaternarios acabados de mencionar. Estos materiales han sido explotados en este lugar, en una antigua gravera.

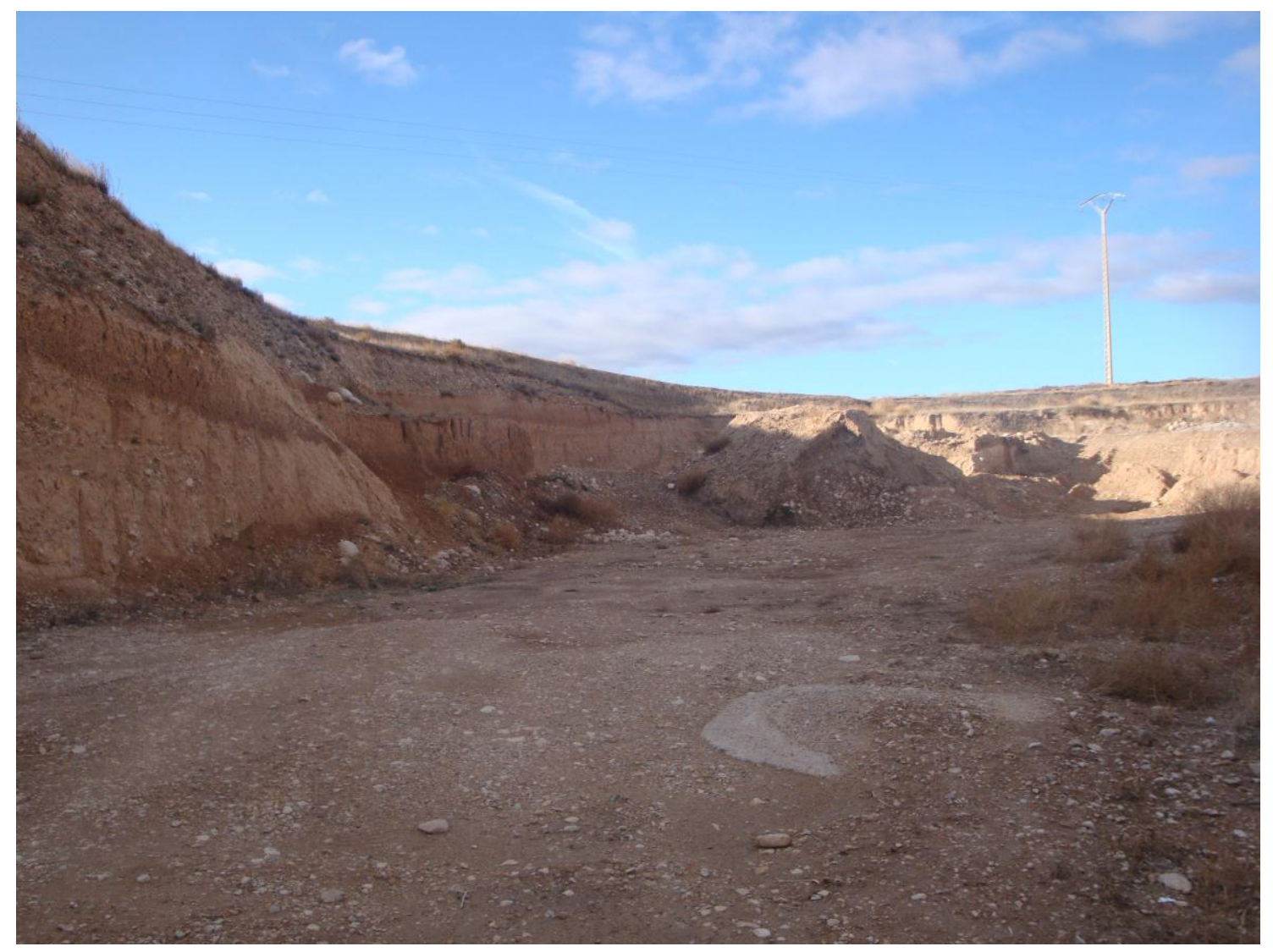

GRAVERA DE LÉCERA

PARADA 2. ESTACIÓN DE VENTAS DE MUNIESA, (término municipal de Muniesa, comarca de Cuencas Mineras). (Hoja 467).

Después de realizar la parada anterior, conviene llegar hasta la carretera procedente de Belchite, la cual conduce hasta Muniesa, entrando momentáneamente en la comarca turolense de Cuencas Mineras. Al llegar a la antigua estación de Ventas de Muniesa, efectuaremos una nueva parada, a unos $11 \mathrm{Km}$ de la anterior.

En este recorrido habremos circulado inicialmente por la Depresión Geológica del Ebro, entre los materiales miocénicos y cuaternarios anteriormente mencionados. Sin embargo, al llegar a las inmediaciones de la Cuesta Blanca, habremos encontrado afloramientos de los materiales carbonatados del Jurásico. Precisamente, estos son 
también los materiales que aparecen en el lugar de la parada, en la estación ferroviaria de las Ventas de Muniesa.

En este lugar, había una estación del Ferrocarril Minero de Utrillas. Este ferrocarril era el lugar por donde se conducía el carbón extraído en las minas de Utrillas, hasta Zaragoza. Constituye un interesante Patrimonio Minero.

PARADA 3 - CONDICIONAL. YESERA DE MONEVA, (término municipal de Moneva, comarca del Campo de Belchite). (Hoja 467).

Después de realizar la parada anterior, conviene continuar por la carretera que conduce a Moneva, partiendo de las Ventas de Muniesa. Por esta ruta, en unos 5 '5 Km llegaremos a Moneva. Luego, ahí convendrá efectuar un breve recorrido hacia el NNW de menos de $0 ` 8 \mathrm{Km}$, para llegar a la yesera. Ahí efectuaremos una nueva parada, a menos de 6'5 Km de la anterior.

En este recorrido, habremos transitado por el Sistema Ibérico, entre afloramientos de los materiales mesozoicos del Triásico, del Jurásico uy del Cretácico. Precisamente, en el lugar de la parada hay un afloramiento de los materiales yesosos del Keuper. Estos, antaño fueron explotados en una pequeña yesera.

PARADA 4. GRAVERA DE PLENAS, (término municipal de Plenas, comarca del Campo de Belchite). (Hoja 467).

Después de realizar la parada anterior, conviene efectuar un recorrido hasta el cercano pueblo de Moyuela. Antes de llegar a ese pueblo encontraremos la carretera de Blesa, que tomaremos hacía ese pueblo, entrando momentáneamente en el municipio de Plenas. A unos $2 \mathrm{Km}$ del cruce de Moyuela y a unos 9 de la parada anterior, efectuaremos la presente.

Este recorrido, lo habremos efectuado también (como el anterior) entre afloramientos de los materiales mesozoicos del Sistema Ibérico. Sin embargo, a menudo estos materiales se hallan recubiertos por terrenos cuaternarios de derrubios de pendiente, como en el lugar de parada. Aquí, estos materiales han sido explotados en una gravera (FOTOGRAFÍA EN LA HOJA SIGUIENTE).

PARADA 5. CANTILES DEL RÍO CÁMARAS, (término municipal de Azuara, comarca del Campo de Belchite). (Hoja 439).

Después de realizar la parada anterior, conviene retroceder hasta el cruce, para ir luego hasta Moyuela. Des de ahí nos convendrá ir hacía Azuara, desde donde continuaremos por la carretera de Herrera de los Navarros. A unos $6 \mathrm{Km}$ de Azuara y a unos 20 de la parada anterior, efectuaremos la presente, frente a los Cantiles del río Cámaras (FOTOGRAFÍA EN LA PÁGINA SIGUIENTE). 


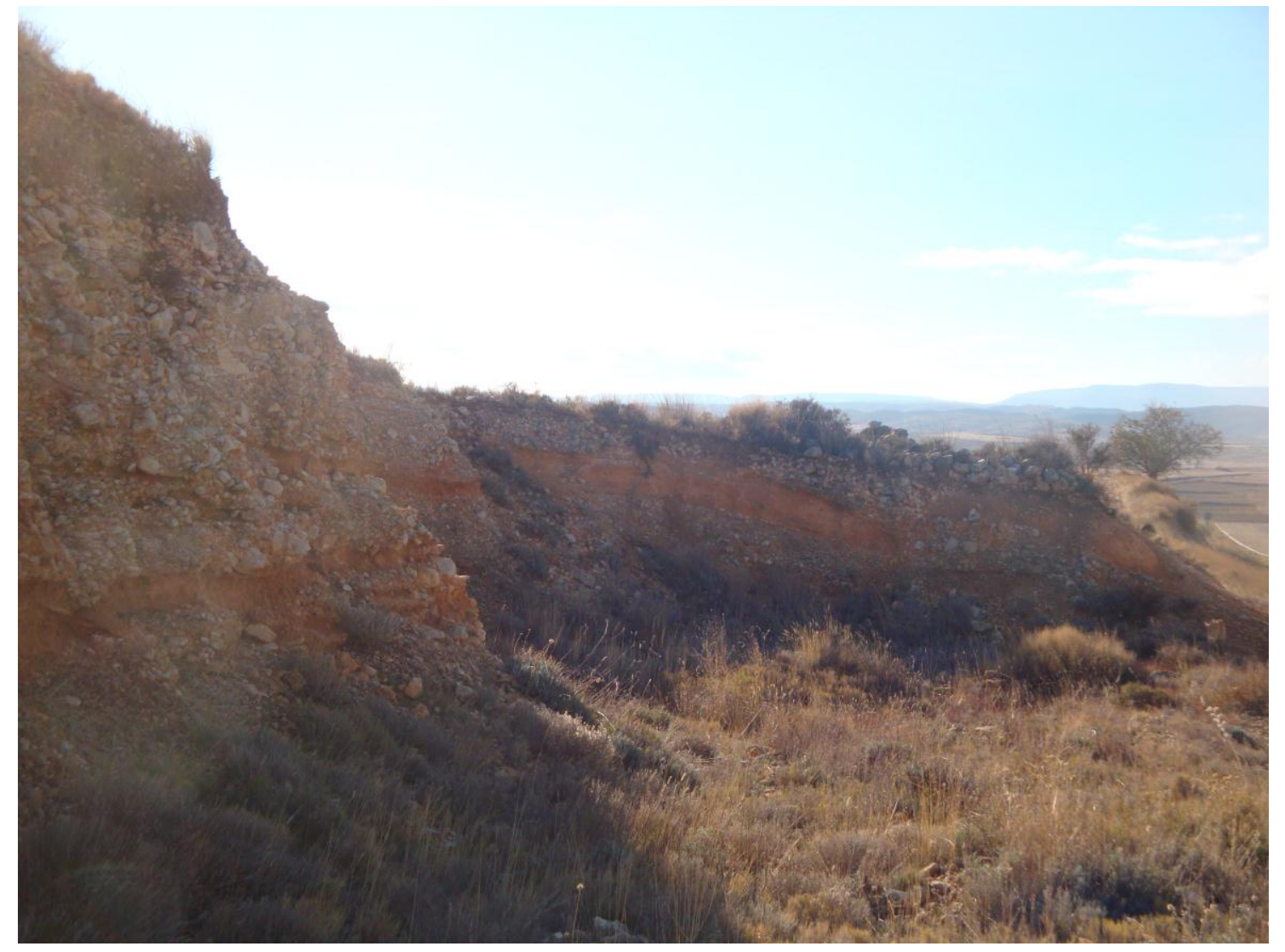

GRAVERA DE PLENAS

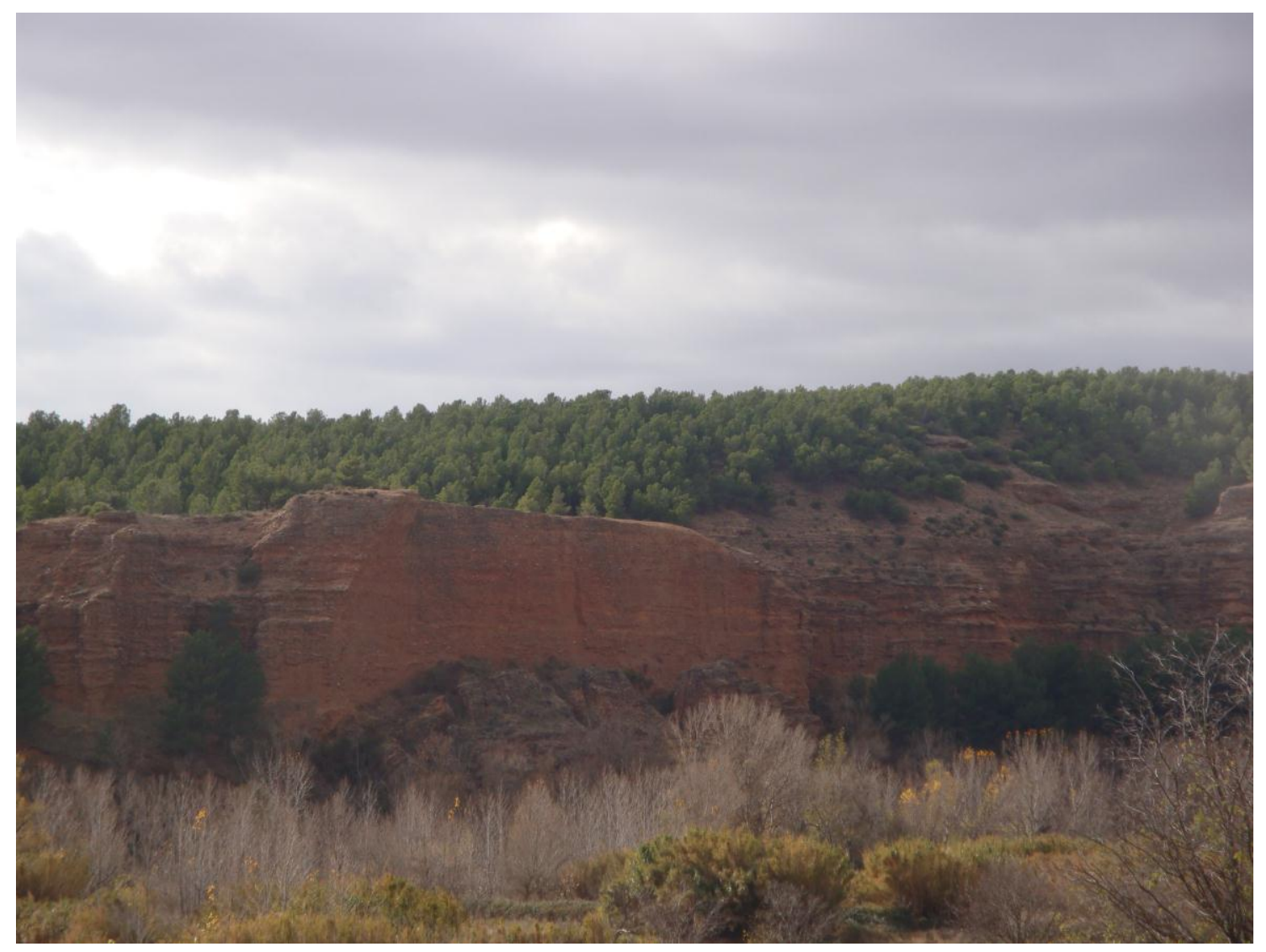

CANTILES DEL RÍO CÁMARAS 
En este recorrido, habremos vuelto a la Depresión Geológica del Ebro, en donde ahora nos encontramos. Precisamente, frente a donde nos hallamos, podemos ver los Cantiles del río Cámaras, formados a partir de la erosión del mencionado río, sobre masas de conglomerados cenozoicos del Mioceno.

PARADA 6. GRAVERA DE AZUARA, (término municipal de Azuara, comarca del Campo de Belchite). (Hoja 439).

Después de realizar la parada anterior, conviene retroceder hasta la población de Azuara, con la intención de continuar el recorrido hacía Fuendetodos. A unos $3 \mathrm{Km}$ de Azuara, y a unos 9 de la parada anterior, efectuaremos la última parada del recorrido de este itinerario.

En este recorrido, realizado íntegramente por la Depresión Geológica del Ebro, habremos visto frecuentes afloramientos de los materiales detríticos cuaternarios, los cuales recubren a los miocénicos de la mencionada depresión.

En este lugar hay un afloramiento de los materiales detríticos cuaternarios, de derrubios de pendiente. Estos materiales han sido explotados en una antigua gravera, totalmente abandonada.

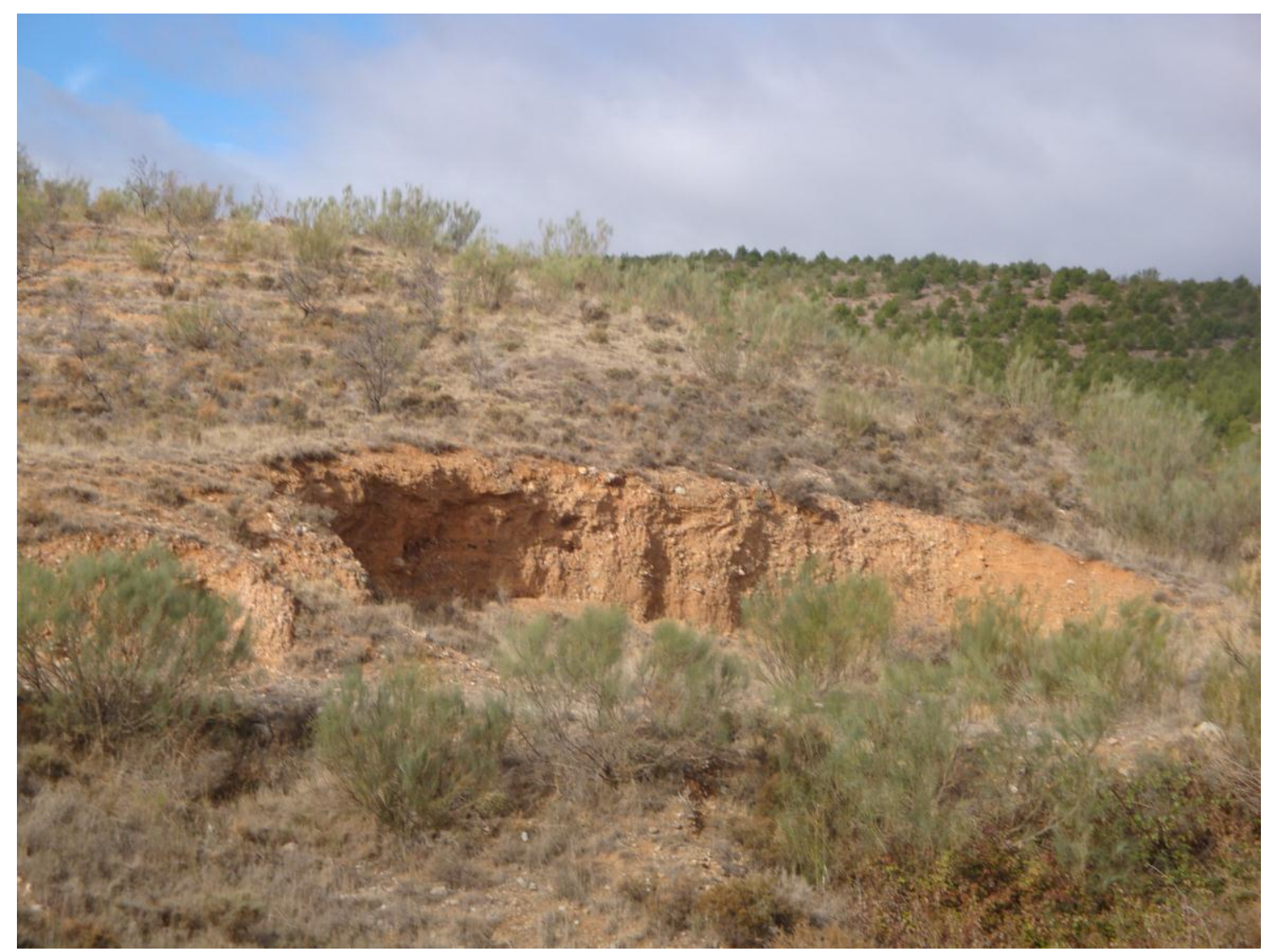

UNA DE LAS GRAVERAS DE AZUARA

EN ESTE LUGAR FINALIZA EL ITINERARIO 


\section{BIBLIOGRAFÍA}

CALVO, M. (2001).- Las Minas de Sal de Remolinos, Zaragoza. Revista de Minerales, Vol. 2, no 2, pp. 3 - 22. Barcelona

CALVO, M. et altri (1988). - Minerales de Aragón, Colección Temas Geológicos, 207 Pág. Zaragoza

GOBIERNO DE ARAGÓN (2001).- Puntos de Interés Geológico de Aragón. Consejería de Medio Ambiente del Gobierno de Aragón. Zaragoza

IGME (1972).- Mapa Geológico de España a escala 1:200.000 (Síntesis de la cartografía existente). Hoja y Memoria nº 32 (Zaragoza). Inst. Geol. Min. España

IGME (1973).- Mapa Metalogenético de España a escala 1:200.000. Hoja y Memoria $n^{\circ} .32$ (Zaragoza). Inst. Geol. Min. España

IGME (1975).- Mapa de Rocas Industriales de España a escala 1:200.000. Hoja y Memoria $n^{\circ} .32$ (Zaragoza). Inst. Geol. Min. España

MAESTRE, A. (1845).- Descripción geognóstica del Distrito Minero de Cataluña y Aragón. Anales de Minas, t. III. Madrid

MATA - PERELLÓ, J.; (1987).- Introducción al conocimiento de las mineralizaciones aragonesas. Mineralogistes de Catalunya, t.III, pp. 258-265. Barcelona

MATA - PERELLÓ, J.M. (1998).- Inventario Mineralógico de la comarca del Campo de Belchite, Rodeno, 25. 22 Pág. Manresa

PRAMES (2005).- Campo de Belchite. Colección RUTASCAL por Aragón. Prames, Gobierno de Aragón. 119 pag. Zaragoza. 